\title{
A MEASURE OF SMOOTHNESS RELATED TO THE LAPLACIAN
}

\author{
Z. DITZIAN
}

Abstract. A $K$-functional on $f \in C\left(R^{d}\right)$ given by

$$
\widetilde{K}\left(f, t^{2}\right)=\inf \left(\|f-g\|+t^{2}\|\Delta g\| ; g \in C^{2}\left(R^{d}\right)\right)
$$

will be shown to be equivalent to the modulus of smoothness

$$
\tilde{w}(f, t)=\sup _{0<h \leq t}\left\|2 d f(x)-\sum_{i=1}^{d}\left[f\left(x+h e_{i}\right)+f\left(x-h e_{i}\right)\right]\right\| .
$$

The situation for other Banach spaces of functions on $R^{d}$ will also be resolved.

\section{INTRODUCTION}

The Laplacian of $f(x), x \in R^{d}$ given by

$$
\Delta f(x)=\frac{\partial^{2}}{\partial x_{1}^{2}} f(x)+\cdots+\frac{\partial^{2}}{\partial x_{d}^{2}} f(x)
$$

was related recently [3] to the discrete Laplacian given by

$$
\widetilde{\Delta}_{h} f(x)=2 d f(x)-\sum_{i=1}^{d}\left(f\left(x+h e_{i}\right)+f\left(x-h e_{i}\right)\right)
$$

where $e_{i}$ is a fixed given orthonormal base of $R^{d}$. It was shown in [3] that for $C\left(R^{d}\right), L_{p}\left(R^{d}\right)$ where $1<p<\infty$ or $L_{1}\left(R^{d}\right)$ the inequality

$$
\left\|h^{-2} \tilde{\Delta}_{h} f\right\| \leq M
$$

(where $M$ is independent of $h$ ) is equivalent to the statement: $\Delta f$ exists in the weak (Sobolev) sense as an element of $L_{\infty}\left(R^{d}\right), L_{p}\left(R^{d}\right), 1<p<\infty$, or $\mathscr{M}\left(R^{d}\right)$ (the space of finite measures) respectively and $\|\Delta f\| \leq M_{1}$.

For a space $B$ of functions on $R^{d}$ on which translation is a continuous isometry, we may define the natural $K$-functional

$$
\widetilde{K}\left(f, t^{2}\right)_{B}=\inf _{g \in S}\left\{\|f-g\|_{B}+t^{2}\|\Delta g\|_{B}\right\}
$$

Received by the editors June 28, 1989.

1980 Mathematics Subject Classification (1985 Revision). Primary 46E35.

Key words and phrases. Laplacian, $K$-functional, smoothness. 
where $g \in S$ means that $\frac{\partial}{\partial x_{i}} g$ and $\left(\frac{\partial}{\partial x_{i}}\right)^{2} g$ exist as strong derivatives in $B$. If the space $B$ is continuously imbedded in $\mathscr{D}^{\prime}$, the space of Schwartz distributions, we define $\widetilde{K}_{*}\left(f, t^{2}\right)_{B}$ by replacing $S$ in (1.4) by $S^{*}$, where $S^{*}$ is the collection of all $g$, such that $\Delta g \in B$ and $\Delta g$ is taken in the weak (distributional) sense. It turns out that $\widetilde{K}\left(f, t^{2}\right)_{B}$ is equivalent to $\widetilde{K}_{*}\left(f, t^{2}\right)_{B}$. The main result of the paper is the equivalence of the $K$-functional $\widetilde{K}$ given by (1.4) with the modulus of smoothness given by

$$
\tilde{\omega}(f, t)_{B}=\sup _{0<h \leq t}\left\|2 d f(x)-\sum_{i=1}^{d}\left[f\left(x+h e_{i}\right)+\dot{f}\left(x-h e_{i}\right)\right]\right\|_{B}
$$

for some given orthonormal base $\left\{e_{i}\right\}_{i=1}^{d}$ of $R^{d}$. That is, it will be shown that

$$
\tilde{\omega}(f, t)_{B} \sim \widetilde{K}\left(f, t^{2}\right)_{B},
$$

which means

$$
C^{-1} \tilde{\omega}(f, t)_{B} \leq \tilde{K}\left(f, t^{2}\right)_{B} \leq C \tilde{\omega}(f, t) .
$$

Proving (1.6), we see that (1.5) is independent of the orthonormal base $\left\{e_{i}\right\}_{i=1}^{d}$. For $L_{p}\left(R^{d}\right)$ the $K$-functional

$$
K_{2}\left(f, t^{2}\right)_{L_{p}\left(R^{d}\right)}=\inf _{g \in s}\left(\|f-g\|_{L_{p}\left(R^{d}\right)}+t^{2} \sum_{i, j}\left\|\frac{\partial^{2}}{\partial x_{i} \partial x_{j}} g\right\|_{L_{p}\left(R^{d}\right)}\right)
$$

where $g$ has distributional derivatives and $\left(\partial^{2} / \partial x_{i} \partial x_{j}\right) g \in L_{p}\left(R^{d}\right)$ was shown to be equivalent to

$$
\omega^{2}(f, t)_{L_{p}\left(R^{d}\right)}=\sup _{0<h \leq t} \sup _{\substack{c \in R^{d} \\|e|=1}}\|f(x+h e)-2 f(x)+f(x-h e)\|_{L_{p}\left(R^{d}\right)}
$$

(see [1, pp. 337-339]). In fact the equivalence between (1.7) and (1.8) is valid with the same proof if $L_{p}\left(R^{d}\right)$ is replaced by a Banach space of functions on $R^{d}$ for which transiation is a continuous isometry and $S$ is replaced by the class of functions for which $\left(\partial^{2} / \partial x_{i} \partial x_{j}\right) g$ is a strong derivative in $B$ or when $B \subset \mathscr{D}^{\prime}$ by the class of functions $g$ for which the distributional derivative $\left(\partial^{2} / \partial x_{i} \partial x_{j}\right) g$ is in $B$.

We should note that $\widetilde{K}\left(f, t^{2}\right)_{C\left(R^{d}\right)}$ and $K_{2}\left(f, t^{2}\right)_{C\left(R^{d}\right)}$ are not equivalent, and also that $\widetilde{K}\left(f, t^{2}\right)_{L_{1}\left(R^{d}\right)}$ and $K_{2}\left(f, t^{2}\right)_{L_{1}\left(R^{d}\right)}$ are not equivalent. It is virtually obvious that $\widetilde{K}\left(f, t^{2}\right)_{L_{p}\left(R^{d}\right)}$ and $K_{2}\left(f, t^{2}\right)_{L_{p}\left(R^{d}\right)}$ are equivalent for $1<$ $p<\infty$. A further relation between $\widetilde{K}\left(f, t^{2}\right)_{B}$ and $K_{2}\left(f, t^{2}\right)_{B}$, and therefore between $\tilde{\omega}(f, t)_{B}$ and $\omega^{2}(f, t)_{B}$, is that the Besov spaces generated by them are equivalent. This means that the $K$-functionals $\widetilde{K}\left(f, t^{2}\right)_{B}$ and $K_{2}\left(f, t^{2}\right)_{B}$ differ only at optimal or near optimal rate. 
As characterization of a given $K$-functional by the behaviour of the function is considered quite a basic problem in interpolation of spaces, $I$ hope the present somewhat combinatorial result will be of interest.

\section{SOME INEQUALITIES}

Throughout this section we will assume that $\left\{e_{i}\right\}_{i=1}^{d}$ is a given fixed orthonormal base of $R^{d}$. We define the operator $\Delta_{v}^{2}$ for $v \in R^{d}$

$$
\Delta_{v}^{2} f(x) \equiv f(x+v)-2 f(x)+f(x-v), \quad \Delta_{v}^{2 l+2} f(x) \equiv \Delta_{v}^{2}\left(\Delta_{v}^{2 l} f(x)\right) .
$$

A Banach space $B$ of functions or distributions on $R^{d}$ is called translation invariant if

$$
\|f(\cdot+v)\|_{B}=\|f(\cdot)\|_{B} \quad \text { for all } v \in R^{d} .
$$

We can now state and prove the following result which will be helpful later.

Theorem 2.1. For a Banach space of functions or distributions on $R^{d}$ which satisfies (2.2), $\Delta_{h i_{i_{j}}}^{2 s_{j}}$ given by (2.1) and $\widetilde{\Delta}_{h}$ given by (1.2) we have

$$
\left\|\sum \Delta_{h e_{i_{1}}}^{2 s_{1}} \cdots \Delta_{h e_{i_{m}}}^{2 s_{m}} f(x)\right\|_{B} \leq C(m, s) \sum_{k=1}^{s_{1}+\cdots+s_{m}=s}\left\|\tilde{\Delta}_{k h} f\right\|_{B}
$$

where the sum on the left of (2.3) is taken on all possibilities $e_{i_{1}} \cdots e_{i_{m}}$ such that $e_{i_{s}} \neq e_{i_{n}}$ for $r \neq s$ and where $e_{i_{j}} \in\left\{e_{i}\right\}_{i=1}^{d}$.

Remark. Note that for $\widetilde{\Delta}_{h} f$ to be defined by (1.2) it is sufficient that $B$ is a Banach space of distribution on $R^{d}$. Observe that in the sum (2.3) two different orders of $e_{i_{1}} \cdots e_{i_{r}}$ are two entries in the sum.

Proof. We first prove our theorem for $m=1$. We can easily obtain the identity

$$
\sum_{i=1}^{d} \Delta_{h e_{i}}^{2 s} f=\sum_{l=0}^{s-1}\left(\begin{array}{c}
2 s \\
l
\end{array}\right)(-1)^{l+1} \tilde{\Delta}_{(s-l) h} f .
$$

This will imply (2.3) for $m=1$. We can now write the identity

$$
\begin{aligned}
& \left(\sum_{i=1}^{d} \Delta_{h e_{i}}^{2 s_{i_{i}}}\right)\left(\sum_{\substack{e_{i_{i} \neq e_{i_{t}}} \\
r \neq t}} \Delta_{h e_{i_{1}}}^{2 s_{1}} \cdots \Delta_{h e_{i_{l-1}}}^{2 s_{l_{l}}}\right) f \\
& =\sum_{\substack{i_{r} \neq i_{t} \\
r \neq t}} \Delta_{h e_{i_{1}}}^{2 s_{1}} \cdots \Delta_{h e_{i_{i}}}^{2 s_{s_{l}}} f+\sum_{j=1}^{l-1} \sum_{\substack{i_{r} \neq i_{t} \\
r \neq t}} \Delta_{h e_{i_{1}}}^{2 s_{s_{1}}} \cdots \Delta_{h e_{i_{j}}}^{2 s_{j}+2 s_{l}} \cdots \Delta_{h e_{t_{l-1}}}^{2 s_{l_{1}}} f .
\end{aligned}
$$


Assuming (2.3) for $m<l$ and utilising (2.2), we have

$$
\begin{aligned}
& \left\|\left(\sum_{i=1}^{d} \Delta_{h e_{i}}^{2 s_{l}}\right) \sum_{\substack{i_{r} \neq i_{t} \\
r \neq t}} \Delta_{h e_{1}}^{2 s_{1}} \cdots \Delta_{h e_{i_{l-1}}}^{2 s_{l-1}} f\right\| \\
& \quad \leq C\left(l-1, s_{1}+\cdots+s_{l-1}\right) 4^{s_{l}} d \sum_{k=1}^{s_{1}+\cdots+s_{l-1}}\left\|\tilde{\Delta}_{k h} f\right\|
\end{aligned}
$$

and

$$
\left\|\sum_{\substack{i_{i} \neq i_{t} \\ r \neq t}} \Delta_{h e_{i_{1}}}^{2 s_{1}} \cdots \Delta_{h e_{i_{j}}}^{2 s_{j}+2 s_{l}} \cdots \Delta_{h e_{i_{l-1}}}^{2 s_{l}-1} f\right\| \leq C\left(l-1, s_{1}+\cdots+s_{l}\right) \sum_{k=1}^{s_{1}+\cdots+s_{l}}\left\|\widetilde{\Delta}_{k h} f\right\|,
$$

which, together with (2.5), implies (2.3) for $m=l$.

We can now prove

Theorem 2.2. For a Banach space $B$ of functions or distributions on $R^{d}$ satisfying (2.2) and $f \in B$ we have

$$
\left\|\Delta_{h e_{i}}^{2 d} f\right\|_{B} \leq C(d) \sum_{k=1}^{d}\left\|\widetilde{\Delta}_{k h} f\right\|_{B}
$$

for $i=1, \ldots, n$.

Proof. We may assume with no loss of generality that $i=1$. We now write

$$
\begin{aligned}
-\Delta_{h e_{1}}^{2}\left(\tilde{\Delta}_{h}\right) & =\Delta_{h e_{1}}^{2}\left(\sum_{i=1}^{d} \Delta_{h e_{i}}^{2}\right)=\Delta_{h e_{1}}^{4}+\Delta_{h e_{1}}^{2} \sum_{i=2}^{d} \Delta_{h e_{i}}^{2} \\
& =\Delta_{h e_{1}}^{4}+\sum_{i \neq j} \Delta_{h e_{i}}^{2} \Delta_{h e_{j}}^{2}-\sum_{\substack{i \neq j \\
i \neq j \\
j \neq 1}} \Delta_{h e_{i}}^{2} \Delta_{h e_{j}}^{2} .
\end{aligned}
$$

Using

$$
\left\|\Delta_{h e_{1}}^{2} \tilde{\Delta}_{h} f\right\| \leq 4\left\|\tilde{\Delta}_{h} f\right\|
$$

and

$$
\left\|\sum_{i \neq j} \Delta_{h e_{i}}^{2} \Delta_{h e_{j}}^{2} f\right\| \leq C(2)\left(\left\|\tilde{\Delta}_{2 h} f\right\|+\left\|\tilde{\Delta}_{h} f\right\|\right),
$$

we have

$$
\left\|\Delta_{h e_{1}}^{4} f-\sum_{\substack{i \neq j \\ i, j \neq 1}} \Delta_{h e_{1}}^{2} \Delta_{h e_{j}}^{2} f\right\| \leq C_{1}(2) \sum_{l=1}^{2}\left\|\tilde{\Delta}_{l h} f\right\| .
$$

As for $d=2$, the set $i \neq j$ and $i, j \neq 1$ is empty, (2.6) is proved for $d=2$. 
We continue and show by induction that

$$
\left\|\Delta_{h e_{1}}^{2 k} f+(-1)^{k-1} \sum_{\substack{i_{i} \neq i_{l} \\ r \neq t \\ i_{r} \neq 1}} \Delta_{h e_{i_{1}}}^{2} \cdots \Delta_{h e_{i_{k}}}^{2} f\right\| \leq C_{1}(k) \sum_{l=1}^{k}\left\|\tilde{\Delta}_{l h} f\right\| .
$$

One can operate on the expression inside the norm on the left of $(2.7)$ to obtain

$$
\begin{aligned}
\Delta_{h e_{1}}^{2}\left(\Delta_{h e_{1}}^{2 k}\right) f+(-1)^{k-1} \Delta_{h e_{1}}^{2} \sum_{\substack{i_{r} \neq i_{t} \\
r \neq t \\
i_{r} \neq 1}} \Delta_{h e_{i_{1}}}^{2} \cdots \Delta_{h e_{i_{k}}}^{2} f \\
=\Delta_{h e_{1}}^{2(k+1)} f+(-1)^{k} \sum_{\substack{i_{r} \neq i_{t} \\
r \neq t \\
i_{r} \neq 1}} \Delta_{h e_{i_{1}}}^{2} \cdots \Delta_{h e_{i_{k+1}}}^{2} f+(-1)^{k-1} \sum_{\substack{i_{r} \neq i_{t} \\
r \neq t}} \Delta_{h e_{i_{1}}}^{2} \cdots \Delta_{h e_{i_{k+1}}}^{2} f .
\end{aligned}
$$

As (2.3) implies

$$
\left\|\sum_{\substack{i_{r} \neq i_{t} \\ r \neq t}} \Delta_{h e_{i_{1}}}^{2} \cdots \Delta_{h e_{i_{k+1}}}^{2} f\right\| \leq C\left(\sum_{l=1}^{k+1}\left\|\tilde{\Delta}_{l h} f\right\|\right),
$$

we have (2.7) for $k+1$. For $k=d$ the sum

$$
\sum_{\substack{i_{r} \neq i_{i} \\ r \neq t \\ i_{r} \neq 1}} \Delta_{h e_{i_{1}}}^{2} \cdots \Delta_{h e_{i_{k}}}^{2}
$$

is a sum on an empty set, and our theorem is proved.

We will now obtain an estimate which, while as stated will be a multivariate result, is in fact a result about functions of one variable.

A Banach space of functions on $R^{d}$ is called strongly continuous if

$$
\|f(\cdot+h e)-f(\cdot)\|_{B}=o(1), \quad h \rightarrow 0 \text { for all unit vectors } e \text { in } R^{d} .
$$

Theorem 2.3. Suppose $B$ is a Banach space of functions on $R^{d}$ for which translation is a strongly continuous isometry, that is (2.2) and (2.8) are satisfied. Then for $0<u<t$ we can find $\alpha_{i}(y) \quad(y \in R)$ fixed even polynomials of degree $2 i$ such that

$$
\left\|\sum_{i=1}^{k-1} \alpha_{i}\left(\frac{u}{t}\right) \Delta_{t e}^{2 i} f-\Delta_{u e}^{2} f\right\|_{B} \leq M \sup _{0<h \leq t}\left\|\Delta_{h e}^{2 k} f\right\|_{B} .
$$

Proof. We define

$$
F(s)=\langle f(\cdot+s e), g(\cdot)\rangle \quad \text { for } g \in B^{*}, s \in R,
$$


where $B^{*}$ is the dual space to $B$. Obviously, $F(s) \in C(R)$. We first show that (2.9) for $F$, that is,

$$
\left\|\sum_{i=1}^{k-1} \alpha_{i}\left(\frac{u}{t}\right) \Delta_{t}^{2 i} F-\Delta_{u}^{2} F\right\|_{C(R)} \leq M \sup _{0<h \leq t}\left\|\Delta_{h}^{2 k} F\right\|_{C(R)}
$$

implies (2.9) in general. Of course, when we deal with $F(s)$ which is a function on $C(R)$, we may write $\Delta_{t}^{2}$ rather than $\Delta_{t e}^{2}$ with $e$ the unit vector in $R=R^{1}$. We choose $g$ such that $\|g\|_{B^{*}}=1$ to obtain

$$
\begin{aligned}
\left|\sum_{i=1}^{k-1} \alpha_{i}\left(\frac{u}{t}\right) \Delta_{t}^{2 i} F(0)-\Delta_{u}^{2} F(0)\right| & \leq\left\|\sum_{i-1}^{k-1} \alpha_{i}\left(\frac{u}{t}\right) \Delta_{t}^{2 i} F-\Delta_{u}^{2} F\right\|_{C(R)} \\
& \leq M \sup _{0<h \leq t}\left\|\Delta_{h}^{2 k} F\right\|_{C(R)} \leq M \sup _{0<h \leq t}\left\|\Delta_{h e}^{2 k} f\right\|_{B} .
\end{aligned}
$$

We can now choose a $g$ (of those that satisfy $\|g\|_{B^{*}}=1$ ) that depends on $u, t, f$ and $\varepsilon>0$ such that

$$
\begin{aligned}
\left\|\sum_{i=1}^{k-1} \alpha_{i}\left(\frac{u}{t}\right) \Delta_{t e}^{2 i} f-\Delta_{h e}^{2} f\right\|_{B}-\varepsilon & \leq\left|\sum_{i=1}^{k-1} \alpha_{i}\left(\frac{u}{t}\right) \Delta_{t}^{2 i} F(0)-\Delta_{u}^{2} F(0)\right| \\
& \leq M \sup _{0<h \leq t}\left\|\Delta_{h e}^{2 k} f\right\|_{B},
\end{aligned}
$$

and as $\varepsilon$ is arbitrary, we complete the proof of (2.9) given (2.10). In fact, we established that the present result is essentially about functions on $R$.

We now construct $\alpha_{i}$ such that the operator

$$
O_{t, u, k} \equiv \sum_{i=1}^{k-1} \alpha_{i}\left(\frac{u}{t}\right) \Delta_{t}^{2 i}-\Delta_{u}^{2}
$$

annihilates polynomials of degree $2 k-1$. It is easy to see, and probably helpful in understanding the direction of the proof, that $\alpha_{1}(u / t)=u^{2} / t^{2}$ and that $\left(u^{2} / t^{2}\right) \Delta_{t}^{2}-\Delta_{u}^{2}$ annihilates polynomials of degree 3 . The construction follows by induction. We assume that we have constructed for $i<l$ even polynomials of degree $2 i, \alpha_{i}(y)$ so that $O_{t, u, l}$ annilhilates polynomials of degree $2 l-1$. As $\Delta_{t}^{2 l}$ also annihilates polynomials of degree $2 l-1$, we construct $\alpha_{l}(y)$ so that at $x=0$ we have

$$
\alpha_{l}\left(\frac{u}{t}\right) \Delta_{t}^{2 l} x^{2 l}=\alpha_{l}\left(\frac{u}{t}\right)(2 l) ! t^{2 l}=-O_{t, u, l} x^{2 l} .
$$

As $O_{t, u, l}$ annihilates polynomials of degrees $2 l-1$, it is sufficient to calculate $O_{t, u, l}(x-a)^{2 l}$ at $a$ or $O_{t, u, l} x^{2 l}$ at 0 (which is equal), and therefore,

$$
-O_{t, u, l} x^{2 l}=2 u^{2 l}-2 \sum_{i=1}^{l-1} \alpha_{i}\left(\frac{u}{t}\right)\left\{\sum_{r=0}^{i}\left(\begin{array}{c}
2 i \\
r
\end{array}\right)(-1)^{r}(i-r)^{2 l}\right\} t^{2 l} \text {. }
$$


As $\alpha_{i}\left(\frac{u}{t}\right)$ is $\frac{-1}{(2 l) !} O_{t, u, l} x^{2 l}$, it is even polynomial in $\left(\frac{u}{t}\right)$ of degree $2 l$, and in fact we can effectively estimate its coefficients. We have now shown that $O_{t, u, l+1}$ annihilates polynomials of degree $2 l$. As $O_{t, u, l+1}(x-a)^{2 l+1}$ at $a$ is equal to zero for all $a$, and as polynomials can be expanded in $(x-a)^{j}$, we have constructed $\alpha_{i}$ as desired.

We now recall (see $[1$, p. 339, Theorem 4.12] for instance) that for any

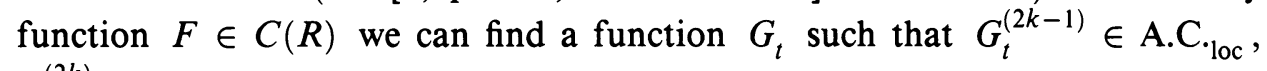
$G_{t}^{(2 k)} \in L_{\infty}$,

$$
\left\|F-G_{t}\right\|_{C(R)} \leq A \sup _{0<h \leq t}\left\|\Delta_{h}^{2 k} F\right\|_{C(R)}
$$

and

$$
t^{2 k}\left\|G_{t}^{(k)}\right\| \leq \sup _{0<h \leq t}\left\|\Delta_{h}^{2 k} F\right\|_{C(R)} .
$$

As the operator $O_{t, u, k}$ is linear, we can estimate it separately on $F-G_{t}$ and $G_{t}$. The estimate of $O_{t, u, k}\left(F-G_{t}\right)$ is trivial since $O_{t, u, k}$ is bounded. The estimate of $O_{t, u, k} G_{t}$ follows from Taylor's formula, the annihilation of $(x-a)^{j}$ for $j<2 k$ and easy computation on the remainder.

In fact, we can prove an analogue of Theorem 2.3 for a Banach space of distribution (generalized functions) on $R^{d}$, that is a space $B \subset \mathscr{D}^{\prime}$ (where $\mathscr{D}^{\prime}$ is the Schwartz space of distributions) such that $B$ is continuously imbedded in $\mathscr{D}^{\prime}$.

Theorem 2.4. Suppose B is a Banach space of distributions which is strongly, weakly or weakly* continuous and on which translations are isometries. Then for the $\alpha_{i}(y) \quad(y \in R)$ of Theorem 2.3, (2.9) is valid.

Proof. We recall that $B$ is weakly continuous if

$$
\langle f(\cdot+v)-f(\cdot), g(\cdot)\rangle=o(1), \quad|v| \rightarrow 0 \text { for all } f \in B \text { and } g \in B^{*}
$$

and weakly* continuous if $B=X^{*}$ and

$$
(2.12)^{\prime}\langle f(\cdot+v)-f(\cdot), g(\cdot)\rangle=o(1), \quad|v| \rightarrow 0 \text { for all } f \in B \text { and } g \in X \text {. }
$$

We now define $F(s)$ as in Theorem 2.3 for $B$ which is strongly or weakly continuous and by

$$
F(s)=\langle f(\cdot+s e), g(\cdot)\rangle \quad \text { for } g \in X, f \in B \text { where } B=X^{*}
$$

for $B$ which is weakly* continuous. We now follow the first part of the proof of Theorem 2.3 to show that $(2.10)$ is sufficient for our result. The assertion (2.10) was proved while proving Theorem 2.3.

\section{THE UPPER ESTIMATE}

We recall the definition $\tilde{K}\left(f, t^{2}\right)_{B}$ and $\tilde{K}_{*}\left(f, t^{2}\right)_{B}$. The $K$-functional $\widetilde{K}\left(f, t^{2}\right)_{B}$ is given by

$$
\widetilde{K}\left(f, t^{2}\right)_{B}=\inf _{g \in S}\left(\|f-g\|_{B}+t^{2}\|\Delta g\|_{B}\right)
$$


where $S$ is the class of elements of $B$ for which the strong first two derivatives exist and are in $B$. The $K$-functional $\widetilde{K}_{*}\left(f, t^{2}\right)_{B}$ is defined for $B \subset \mathscr{D}^{\prime}$ ( $B$ is continuously imbedded in the Schwartz space of distributions $\mathscr{D}^{\prime}$ ) with $S^{*}$ replacing $S$, and $S^{*}$ is the class of elements of $\mathscr{D}^{\prime}$ for which the weak Laplacian belongs to $B$. We note that it is possible that $\widetilde{K}\left(f, t^{2}\right)=O\left(t^{2}\right)$ and at the same time $\left(\partial / \partial x_{i}\right)^{2} f$ is not in $B$ or does not exist even locally.

Theorem 3.1. Suppose $f \in B$, translations on $B$ are isometries and the expressions $\widetilde{K}\left(f, t^{2}\right)_{B}$ and $\tilde{\omega}(f, t)_{B}$ are given by (1.4) and (1.5) respectively. Then we have for a Banach space $B$ over $R^{d}$ for which translations are strongly continuous

$$
\widetilde{K}\left(f, t^{2}\right)_{B} \leq C \tilde{\omega}(f, t)_{B} .
$$

If $B \subset \mathscr{D}^{\prime}$ and translations on $B$ are strongly, weakly or weakly* continuous, then

$$
\widetilde{K}_{*}\left(f, t^{2}\right)_{B} \leq C \tilde{\omega}(f, t)_{B} .
$$

Proof. Using the definitions of $\widetilde{K}\left(f, t^{2}\right)$ and $\widetilde{K}_{*}\left(f, t^{2}\right)$,

$$
\widetilde{K}\left(f,(a t)^{2}\right) \leq a^{2} \widetilde{K}\left(f, t^{2}\right) \text {, and } \widetilde{K}_{*}\left(f,(a t)^{2}\right) \leq a^{2} \widetilde{K}_{*}\left(f, t^{2}\right) \quad \text { for } a>1 .
$$

As $d$ is a fixed finite integer, it is sufficient to prove

$$
\tilde{K}\left(f, t^{2}\right) \leq C \tilde{\omega}\left(f, t d^{2}\right) \quad \text { and } \quad \widetilde{K}_{*}\left(f, t^{2}\right) \leq C \tilde{\omega}\left(f, t d^{2}\right) .
$$

To prove (3.3) we construct the function

$$
g_{t}(x)=t^{-2 d} \int_{-t / 2}^{t / 2} \cdots \int_{-t / 2}^{t / 2} f\left(x+\left(u_{1}+u_{2}\right) e_{1}+\cdots+\left(u_{2 d-1}+u_{2 d}\right) e_{d}\right) d u_{1} \cdots d u_{2 d}
$$

and show that

$$
\left\|g_{t}-f\right\| \leq C \tilde{\omega}\left(f, t d^{2}\right)
$$

and

$$
\left\|\Delta g_{t}\right\| \leq C t^{-2} \tilde{\omega}\left(f, t d^{2}\right) .
$$

In (3.6) $\Delta g_{t}$ is taken as a sum of strong derivatives for the estimate of $\widetilde{K}\left(f, t^{2}\right)_{B}$ and a weak distributional derivative for the estimate of $\widetilde{K}_{*}\left(f, t^{2}\right)_{B}$.

We prove (3.5) first. For $1 \leq l \leq d$ we denote

$$
\begin{aligned}
I(f, l) & \equiv I\left(f, l, e_{i_{1}}, \ldots, e_{i_{d}}\right) \\
& \equiv t^{-2 d+2 l-2} \int_{-t / 2}^{t / 2} \cdots \int_{-t / 2}^{t / 2} f\left(x+\sum_{j=l}^{d}\left(u_{2 j-1}+u_{2 j}\right) e_{i_{j}}\right) d u_{2 l-1} \cdots d u_{2 d},
\end{aligned}
$$

$I(f, d+1) \equiv f(x)$. 
We may now write

$$
g_{t}(x)-f(x)=\frac{1}{d !} \sum \sum_{l=1}^{d}[I(f, l)-I(f, l+1)]
$$

where the first sum indicates summation on all permutations of $e_{1} \cdots e_{d}$. Therefore, it is sufficient to estimate

$$
\sum(I(f, l)-I(f, l+1))
$$

for a fixed $l, 1 \leq l \leq d$, where the sum is taken on all permutations of $e_{1} \cdots e_{d}$. We can write

$$
\begin{aligned}
I(f, l) & -I(f, l+1) \\
= & t^{-2} \int_{0}^{t / 2} \int_{0}^{t / 2} \Delta_{\left(u_{2 l-1}+u_{2 l}\right) e_{i_{l}}}^{2} I(f, l+1) d u_{2 l-1} d u_{2 l} \\
= & t^{-2} \int_{0}^{t / 2} \int_{0}^{t / 2} \Delta_{\left(u_{2 l-1}+u_{2 l}\right) e_{i_{l}}}^{2} f(x) d u_{2 l-1} d u_{2 l} \\
& +\sum_{k=l+1}^{d} t^{-2} \int_{0}^{t / 2} \int_{0}^{t / 2} \Delta_{\left(u_{2 l-1}+u_{2 l}\right) e_{i_{l}}}^{2}(I(f, k)-I(f, k+1)) d u_{2 l-1} d u_{2 l} \\
= & \left.\sum_{\left\{l_{i}\right\}_{i=1}^{m} \subset\{j\}_{l}^{d}, l_{1}=l} t^{-2 m}\right\} \\
& \times \int_{0}^{t / 2} \cdots \int_{0}^{t / 2}\left\{\prod_{k=1}^{m} \Delta_{\left(u_{2 k-1}+u_{2 k}\right) e_{l_{k}}}^{2} \ldots f(x) d u_{1} \cdots d u_{2 m} .\right.
\end{aligned}
$$

To summarize,

$$
\begin{aligned}
g_{t}(x)-f(x)= & \frac{1}{d} t^{-2} \int_{0}^{t / 2} \int_{0}^{t / 2} \widetilde{\Delta}_{\left(u_{1}+u_{2}\right)} f(x) d u_{1} d u_{2} \\
& +\frac{1}{d !} \sum^{*} \sum_{m=2}^{d} t^{-2 m} \int_{0}^{t / 2} \cdots \int_{0}^{t / 2} \\
& \times\left\{\sum^{* *} \Delta_{\left(u_{1}+u_{2}\right) e_{j_{1}}}^{2} \cdots \Delta_{\left(u_{2 m-1}+u_{2 m}\right) e_{j_{m}}}^{2} f(x)\right\} d u_{1} \cdots d u_{2 m} \\
\equiv & I+J
\end{aligned}
$$

where $\sum^{*}$ is the sum on all permutations of $\left\{e_{i}\right\}$, and $\sum^{* *}$ is the sum on all possible collections $e_{j_{1}} \cdots e_{j_{m}}$ such that $e_{j_{r}} \in\left\{e_{j}^{*}\right\}$, (where $\left\{e_{j}^{*}\right\}$ is the given permutation) and $e_{j_{r}} \neq e_{j_{s}}$ for $r \neq s$. The estimate of $J$ is obvious. For the estimate of $I$ we will need the results in Theorems 2.2 and 2.3.

To estimate $I$ it is clear that we have only to estimate terms of the type

$$
\sum \Delta_{\left(u_{1}+u_{2}\right) e_{j_{1}}}^{2} \cdots \Delta_{\left(u_{2 m-1}+u_{2 m}\right) e_{j_{m}}}^{2} f(x)
$$

where the sum is taken on all sequences of $m$ different vectors $e_{j_{1}} \cdots e_{j_{m}}$ such that $e_{j_{l}} \in\left\{e_{i}\right\}_{i=1}^{d}$ and $u_{i}$ satisfy $u_{i} \geq 0$ and $u_{2 j-1}+u_{2 j} \leq t$. We can write the 
expression in (3.8) as

$$
\sum \Delta_{v_{1} e_{j_{1}}}^{2} \cdots \Delta_{v_{m} e_{j_{m}}}^{2} f(x)
$$

with $0<v_{i} \leq t$. Following Theorems 2.3 and 2.4 , we have

$$
\begin{aligned}
& \sum \Delta_{v_{1} e_{j_{l}}}^{2} \cdots \Delta_{v_{m} e_{j_{m}}}^{2} f(x) \\
& =(-1)^{m} \sum \prod_{l=1}^{m}\left(\sum_{i=1}^{d-1} \alpha_{i}\left(\frac{v_{l}}{t}\right) \Delta_{t e_{j_{l}}}^{2 i}-\Delta_{v_{l} e_{j_{l}}}^{2}\right) f(x) \\
& \quad+(-1)^{m+1} \sum \prod_{l=1}^{m} \sum_{i=1}^{d-1} \alpha_{i}\left(\frac{v_{l}}{t}\right) \Delta_{t e_{j_{l}}}^{2 i} f(x)+R \\
& \equiv S+L+R
\end{aligned}
$$

where $R$ is a finite sum of translations of

$$
\sum_{i=1}^{d-1} \alpha_{i}\left(\frac{v_{l}}{t}\right) \Delta_{t e_{j_{l}}}^{2 i}-\Delta_{v_{l} e_{j_{l}}}^{2} f(x)
$$

multiplied by finite products of $\alpha_{i}\left(v_{j} / t\right)$, and $\alpha_{i}\left(v_{j} / t\right)$ are those given in Theorems 2.3 and 2.4, and therefore, bounded for $0 \leq v_{j} \leq t$. We now use Theorems $2.3,2.4$ and 2.2 to obtain

$$
\begin{gathered}
\left\|\left\{\sum_{i=1}^{d_{1}} \alpha_{i}\left(\frac{v_{l}}{t}\right) \Delta_{t e_{j_{l}}}^{2 i}-\Delta_{v_{l} e_{j_{l}}}^{2}\right\} f\right\| \leq M \sup _{0<h \leq t}\left\|\Delta_{h e_{j_{l}}}^{2 d} f\right\| \\
\leq M_{1} \sup _{0<h \leq t} \sum_{k=1}^{d}\left\|\tilde{\Delta}_{k h} f\right\| \leq M_{2} \tilde{\omega}(f, t d) .
\end{gathered}
$$

The inequality (3.9) yields the appropriate estimate for $S$ and $R$.

To estimate $L$ we have to estimate terms like

$$
\sum \Delta_{t e_{j_{1}}}^{2 i_{l}} \cdots \Delta_{t e_{j_{m}}}^{2 i_{m}} f(x)
$$

where the sum is taken to all subcollections of different $e_{j_{1}} \cdots e_{j_{m}}$, and therefore, using Theorem 2.1,

$$
\begin{aligned}
\left\|\sum \Delta_{t e_{\jmath_{1}}}^{2 i_{1}} \cdots \Delta_{t e_{m}}^{2 i_{m}} f\right\| & \leq M_{2} \sum_{k=1}^{i_{1}+\cdots+i_{m}}\left\|\tilde{\Delta}_{k t} f\right\| \\
& \leq M_{3} \tilde{\omega}(f,(d-1) m t) \\
& \leq M_{3} \tilde{\omega}\left(f, d^{2} t\right) .
\end{aligned}
$$


To prove the second crucial inequality, i.e. the estimate (3.6), we write (3.10)

$$
\begin{aligned}
\Delta g_{t}= & t^{-2} \sum_{i=1}^{d} \Delta_{t_{e_{i}}} t^{-2 d+2} \\
& \times\left\{\int_{-t / 2}^{t / 2} \cdots \int_{-t / 2}^{t / 2} f\left(x+\sum_{l \neq i}\left(u_{2 l-1}+u_{2 l}\right) e_{l}\right) d u_{1} \cdots d u_{2 i-2}\right. \\
& \left.\times d u_{2 i+1} \cdots d u_{2 d}\right\} .
\end{aligned}
$$

The expression (3.10) for $\Delta g_{t}$ follows as a strong derivative of $g_{t}$ in case $B$ is strongly continuous and as a weak (distributional) derivative for $B \subset \mathscr{D}^{\prime}$.

We now follow earlier considerations to obtain

$$
\left.\begin{array}{rl}
\Delta g_{t}= & t^{-2} \sum_{i=1}^{d} \Delta_{t e_{i}}^{2} f(x)+\frac{1}{d !} t^{-2} \sum_{i=1}^{d} \Delta_{t e_{i}}^{2} \\
& \times\left\{\sum_{e_{j_{l}} \neq e_{i}}^{*} t^{-2 d+2} \int_{-t / 2}^{t / 2} \cdots \int_{-t / 2}^{t / 2} f\left(x+\sum_{r=1}^{d-1}\left(u_{2 r-1}+u_{2 r}\right) e_{j_{r}}\right)\right. \\
\times d u_{1} \cdots d u_{2 d-2}-f(x)
\end{array}\right\}
$$

where the sum $\sum^{*}$ is taken on all permutations of $e_{j}$ with $j \neq i$. The estimate (3.6) now follows the estimate used for (3.5) with the change that here we have to estimate

$$
t^{-2} \sum \Delta_{t e_{j_{1}}}^{2} \Delta_{\left(u_{1}+u_{2}\right) e_{j_{2}}}^{2} \cdots \Delta_{\left(u_{2 m-3}+u_{2 m-2}\right) e_{j_{m}}}^{2}
$$

for $m>1$ and the sum is on all $j_{1} \cdots j_{m}$ such that $e_{j_{k}} \in\left\{e_{i}\right\}_{i=1}^{d}$ and $e_{j_{r}} \neq e_{j_{s}}$ for $r \neq s$ rather than (3.8). The fact that the first term is already $\Delta_{t e_{j_{1}}}^{2}$ does not make much difference to the proof which follows that of (3.8) but leads to somewhat smaller bounds.

\section{THE LOWER ESTIMATE}

We now estimate $\tilde{\omega}$ by $\widetilde{K}$ or $\widetilde{K}_{*}$ to complete the proof of the equivalence of $\tilde{\omega}$ with these $K$-functionals. We first prove the result for $B=C\left(R^{d}\right)$.

Theorem 4.1. For $f \in C\left(R^{d}\right)$

$$
\tilde{\omega}(f, t)_{C\left(R^{d}\right)} \leq C \tilde{K}\left(f, t^{2}\right)_{C\left(R^{d}\right)} .
$$

Remark. For the sake of Theorem 4.1 the infimum in the definition of $\widetilde{K}$ (see (1.4)) is on all $g$ such that $\Delta g$ exists in the strong sense and is in $C\left(R^{d}\right)$. 
However. as we will see later, existence can be taken in the weak (Sobolev) sense or weak* sense and in $L_{\infty}\left(R^{d}\right)$ with no effect on (4.1).

Proof. From the definition of $\tilde{K}\left(f, t^{2}\right)_{C\left(R^{d}\right)}$ it follows that a function $g_{t} \in$ $C\left(R^{d}\right)$ exists satisfying

$$
\left\|f-g_{t}\right\|_{B} \leq 2 \widetilde{K}\left(f, t^{2}\right)_{B}
$$

and

$$
t^{2}\left\|\Delta g_{t}\right\|_{B} \leq 2 \widetilde{K}\left(f, t^{2}\right)_{B}
$$

for $B=C\left(R^{d}\right)$ where $\Delta g_{t}$ is the strong derivative in $C\left(R^{d}\right)$. Obviously,

$$
\tilde{\omega}(f, t)_{B} \leq \tilde{\omega}\left(f-g_{t}, t\right)_{B}+\tilde{\omega}\left(g_{t}, t\right)_{B}
$$

for $B=C\left(R^{d}\right)$ as well as for other $B$. For any $g_{t}$ satisfying (4.2) we have

$$
\omega\left(f-g_{t}, t\right)_{B} \leq 4 d\left\|f-g_{t}\right\|_{B} \leq 8 d \tilde{K}\left(f, t^{2}\right)_{B} .
$$

To estimate $\omega\left(g_{t}, t\right)_{C\left(R^{d}\right)}$ we use the result of [3, p. 112] and write

$$
\begin{aligned}
\tilde{\omega}\left(g_{t}, t\right)_{C\left(R^{d}\right)} & \leq \sup _{0<h \leq t}\left\|\widetilde{\Delta}_{h} g_{t}\right\|_{C\left(R^{d}\right)} \\
& \leq t^{2} \sup _{0 \leq h \leq t}\left\|h^{-2} \widetilde{\Delta}_{h} g_{t}\right\|_{C\left(R^{d}\right)} \leq M t^{2}\left\|\Delta g_{t}\right\|_{C\left(R^{d}\right)},
\end{aligned}
$$

which concludes the proof of our theorem.

We can now deduce the lower estimate, for other spaces $B$, of $\widetilde{K}\left(f, t^{2}\right)_{B}$ as well as of $\widetilde{K}_{*}\left(f, t^{2}\right)_{B}$.

Theorem 4.2. Suppose $f \in B$, translation on $B$ are isometries and the expressions $\widetilde{K}\left(f, t^{2}\right)_{B}$ and $\tilde{\omega}(f, t)_{B}$ are given by (1.4) and (1.5) respectively. Then for a Banach space for which translations are strongly continuous

$$
\tilde{\omega}(f, t)_{B} \leq A \tilde{K}\left(f, t^{2}\right)_{B}
$$

and for a Banach space $B, B \subset \mathscr{D}^{\prime}$, for which translations are weakly continuous and $\mathscr{D}$ dense in $B^{*}$ or weakly* continuous and $\mathscr{D}$ dense in $X\left(X^{*}=B\right)$

$$
\tilde{\omega}(f, t)_{B} \leq A \widetilde{K}_{*}\left(f, t^{2}\right)_{B} .
$$

Proof. We follow the proof of Theorem 4.1. From the definitions of $\widetilde{K}$ and $\widetilde{K}_{*}$ we have a function $g_{t}$ satisfying

$$
\left\|f-g_{t}\right\|_{B} \leq 2 \tilde{K}\left(f, t^{2}\right)_{B} \quad \text { or } \quad\left\|f-g_{t}\right\|_{B} \leq 2 \tilde{K}_{*}\left(f, t^{2}\right)_{B}
$$

and

$$
t^{2}\left\|\Delta g_{t}\right\| \leq 2 \widetilde{K}\left(f, t^{2}\right)_{B} \quad \text { or } \quad\left\|\Delta g_{t}\right\|_{B} \leq 2 \widetilde{K}_{*}\left(f, t^{2}\right)_{B} .
$$

This implies (4.5) for the first step in the proof of (4.8) or with $\widetilde{K}_{*}\left(f, t^{2}\right)_{B}$ replacing $\widetilde{K}\left(f, t^{2}\right)_{B}$ for the corresponding step in the proof of (4.9). To continue with the proof of (4.8) we write

$$
G_{t}(x) \equiv\left\langle g_{t}(\cdot+x), \varphi(\cdot)\right\rangle, \quad \varphi \in B^{*},\|\varphi\|_{B^{*}} \leq 1,
$$


and therefore, $\Delta G_{t}$ exists in the sense of strong derivatives in $C\left(R^{d}\right)$ and it satisfies

$$
\left\|\Delta G_{t}\right\|_{C\left(R^{d}\right)} \leq\left\|\Delta g_{t}\right\|_{B}
$$

This implies for $0<h \leq t$

$$
\left\|\widetilde{\Delta}_{h} G_{t}\right\|_{C\left(R^{d}\right)} \leq M t^{2}\left\|\Delta G_{t}\right\|_{C\left(R^{d}\right)} \leq M t^{2}\left\|\Delta g_{t}\right\|_{B}
$$

with $M$ that is independent of $t$ or $\varphi$. Therefore, for $0<h \leq t$

$$
\left\|\widetilde{\Delta}_{h} g_{t}\right\| \leq M t^{2}\left\|\Delta g_{t}\right\|_{B} \leq 2 M t^{2} \tilde{K}\left(f, t^{2}\right)_{B},
$$

which completes the proof of $(4.8)$.

To continue with the proof of $(4.9)$ we define again

$$
G_{t}(x) \equiv\left\langle g_{t}(\cdot+x), \varphi(\cdot)\right\rangle
$$

with $\varphi \in B^{*}$ when translations are weakly continuous and $\mathscr{D}$ is dense in $B^{*}$ and with $\varphi \in X$ when translations are weakly* continuous on $B=X^{*}$ and $\mathscr{D}$ is dense in $X$. We now complete the proof of (4.9) following the steps used earlier for the proof of $(4.8)$.

\section{REMARKS}

Remark 5.1. We obtain the $K$-functional $\tilde{K}^{* *}\left(f, t^{2}\right)_{B}$ by replacing $S$ of $\widetilde{K}\left(f, t^{2}\right)_{B}$ by $S^{* *}$ where $S^{* *}$ is the collection of all the functions $g$ for which $\Delta g$ exists in the weak or weak* sense and $B$ is such that translations are weakly or weakly* continuous respectively. This $K$-functional is also equivalent to $\tilde{\omega}(f, t)_{B}$.

Remark 5.2. If for $L_{p}\left(R^{d}\right), 1 \leq p \leq \infty$, we define $S^{* * *}$ by

$$
S^{* * *}=\left\{g \in L_{p}\left(R^{d}\right) ; \frac{\partial}{\partial u} g\left(x+h e_{i}\right) \in \text { A.C. }_{\text {loc }} \text { and } \Delta g \in L_{p}\left(R^{d}\right)\right\},
$$

the corresponding $K$-functional, $K_{* * *}\left(f, t^{2}\right)_{p}$, is equivalent to $\tilde{\omega}(f, t)_{p}$.

Remark 5.3. From the above results and those in earlier sections we see that for some spaces the $K$-functionals $\widetilde{K}\left(f, t^{2}\right), \quad \widetilde{K}_{*}\left(f, t^{2}\right), \quad \widetilde{K}_{* *}\left(f, t^{2}\right)$ and $\widetilde{K}_{* * *}\left(f, t^{2}\right)$ are defined and are equivalent. In fact in $L_{p}\left(R^{d}\right)$ and in other situations they are equal.

Remark 5.4. The $K$-functionals $\widetilde{K}\left(f, t^{2}\right)_{B}$ and $\widetilde{K}_{*}\left(f, t^{2}\right)_{B}$ are independent of the orthogonal system $\left\{e_{i}\right\}$, and therefore, so is the corresponding $\tilde{\omega}(f, t)_{B}$.

Remark 5.5. For $1 \leq p \leq \infty$ one usually investigates the $K$-functional

$$
K_{2}\left(f, t^{2}\right)_{p}=\inf _{g \in A}\left(\|f-g\|+t^{2} \sum_{i, j}\left\|\frac{\partial^{2}}{\partial x_{i} \partial x_{j}} g\right\|_{p}\right)
$$


where $A$ is the class for which $\partial^{2} g / \partial x_{i} \partial x_{j} \in L_{p}$ (and the derivatives are taken in the Sobolev sense). For $1<p<\infty, \widetilde{K}\left(f, t^{2}\right)_{p}$ and $K_{2}\left(f, t^{2}\right)_{p}$ are equivalent as

$$
\left\|\frac{\partial^{2}}{\partial x_{i} \partial x_{j}} g\right\|_{p} \leq C(p)\|\Delta g\|_{p} \quad(\text { for } 1<p<\infty) .
$$

Remark 5.6. For $p=\infty$ and $p=1, K_{2}\left(f, t^{2}\right)_{p}$ is no longer equivalent to $\widetilde{K}\left(f, t^{2}\right)_{p}$; otherwise the inequality

$$
\left\|\frac{\partial^{2} f}{\partial x \partial y}\right\|_{p} \leq C\|\Delta f\|_{p}
$$

which is valid only for $1<p<\infty$, would be valid for $p=1$ and $p=\infty$ as well, and this is not true. For $p=\infty$ an example can be given that shows that $K_{2}\left(f, t^{2}\right)_{\infty}$ is not equivalent to $\widetilde{K}\left(f, t^{2}\right)_{\infty}$ (see for instance [2]).

Remark 5.7. In the next section we will compare Besov space induced by $\widetilde{K}\left(f, t^{2}\right)_{B}$ and $K_{2}\left(f, t^{2}\right)_{B}$ and find them equivalent, and hence only classes of functions with optimal or near optimal behaviour are different.

\section{BESOV TYPE SPACES}

For a given $K$-functional the related Besov space is given by its norm

$$
\begin{gathered}
\|f\|_{B_{q}^{n}}=\left\{\int_{0}^{1}\left(t^{-\alpha} K(f, t)\right)^{q} \frac{d t}{t}\right\}^{1 / q}, \quad 1 \leq q<\infty . \\
\|f\|_{B_{\infty}^{n}}=\sup _{t} t^{-\alpha} K(f, t) .
\end{gathered}
$$

Generally, an additional index $p$ is given to indicate that the $K$ functional is between the space $L_{p}$ and a subspace of $L_{p}$. However, we allow here a general class of spaces $B$ rather than $L_{p}$ to be the underlying space for our $K$-functional.

We define the spaces $\widetilde{B}_{q}^{\alpha}$ and $\bar{B}_{q}^{\alpha}$ as the Besov spaces introduced by (6.1) with the $K$-functionals $\widetilde{K}\left(f, t^{2}\right)_{B}$ and $K_{2}\left(f, t^{2}\right)_{B}$ respectively.

We can now state and prove the following equivalence theorem.

Theorem 6.1. For $1 \leq q \leq \infty$ and $0<\alpha<2$ the spaces $\widetilde{B}_{q}^{\alpha}$ and $\bar{B}_{q}^{\alpha}$ are equivalent.

Proof. For $\alpha<2, \bar{B}_{q}^{\alpha}$ is clearly equivalent to $B_{q}^{\alpha}$ given by

$$
\|f\|_{B_{q}^{n}}=\left\{\begin{array}{l}
\left\{\int_{0}^{1}\left(t^{-\alpha} \omega^{-2 d}(f, t)_{B}\right)^{q} \frac{d t}{t}\right\}^{1 / q}, \quad 1 \leq q \leq \infty, \\
\sup _{t} t^{-\alpha} \omega^{2 d}(f, t)_{B}, \quad q=\infty,
\end{array}\right.
$$

where

$$
\omega^{2 d}(f, t)_{B}=\sup _{\substack{0<h \leq t \\ e \in S}}\left\|\Delta_{h e}^{2 d} f\right\|_{B}
$$

as it is valid also for any integer $r$ replacing $2 d$ in (6.2). 
Using Theorem 2.2, it follows that

$$
\omega^{2 d}(f, t)_{B} \leq C \tilde{K}\left(f, d^{2} t\right)
$$

since the direction $e_{i}$ given in that theorem is immaterial because the Laplacian can be expressed in different coordinates. Therefore,

$$
\|f\|_{B_{q}^{\alpha}} \leq C\|f\|_{\bar{B}_{q}^{\alpha}} \leq C_{1}\|f\|_{\bar{B}_{q}^{\alpha}} \leq C_{2}\|f\|_{B_{q}^{\alpha}} .
$$

\section{ON AN A PRIORI ESTIMATE}

A priori estimates are discussed in many articles (see for instance [4]) and we would like to note that the present result makes a small contribution in this direction.

Theorem 7.1. Suppose $B$ is a Banach space for which translations are isometries on $R^{d}$, and $B \subset \mathscr{D}^{\prime}$ (where $\mathscr{D}^{\prime}$ is the space of distributions) translations are strongly continuous on $B$ and $\mathscr{D}$ is dense in the dual to $B$ or in the predual of $B$. Then $\Delta f$ exists in $\mathscr{D}^{\prime}$ and $\Delta f \in \operatorname{Lip}(\alpha, B), 0<\alpha<1$, implies $f \in \operatorname{Lip}^{*}(2+\alpha, B)$.

Remark 7.2. There are other analogues of Theorem 7.1, but I believe the present form will be of use. The condition $0<\alpha<1$ in Theorem 7.1 can be replaced by any $\beta>0$ such that $[\beta] \neq \beta$. Recall that $g \in \operatorname{Lip}^{*}(\beta, B)$ means

$$
\left\|\Delta_{h}^{r} g\right\| \leq M h^{\beta} \quad \text { for } r>\beta .
$$

Proof. We can write

$$
\left\|\Delta \Delta_{h e} f\right\|_{B}=\left\|\Delta_{h e} \Delta f\right\|_{B} \leq C h^{\alpha} .
$$

Using [3], we have

$$
\left\|\eta^{-2} \widetilde{\Delta}_{\eta} g\right\| \leq M\|\Delta g\|
$$

and therefore,

$$
\left\|\eta^{-2} \widetilde{\Delta}_{\eta} \Delta_{h e} f\right\| \leq\left\|\Delta_{h e} \Delta f\right\|
$$

Using Theorem 2.2, we have

$$
\begin{aligned}
\left\|\Delta_{h e}^{2 d} \Delta_{h e} f\right\| & \leq C \sum_{j=1}^{d}\left\|\widetilde{\Delta}_{j h} \Delta_{h e} f\right\| \\
& \leq C_{1} h^{2} \sum_{j=1}^{d^{2}}\left\|(j h)^{-2} \widetilde{\Delta}_{j h} \Delta_{h e} f\right\| \\
& \leq C_{2} h^{2} \sup _{\eta}\left\|\eta^{-2} \widetilde{\Delta}_{\eta} \Delta_{h e} f\right\| \\
& \leq C_{2} h^{2+\alpha},
\end{aligned}
$$

which concludes the proof of our theorem. 


\section{REFERENCES}

1. C. Bennett and R. Sharpley, Interpolation of operators, Academic Press, 1988.

2. Z. Ditzian, Moduli of continuity in $R^{n}$ and $D \subset R^{n}$, Trans. Amer. Math. Soc. 282 (1984), 611-623.

3. __ The Laplacian and the discrete Laplacian, Compositio Math. 69 (1989), 111-120.

4. R. S. Sharpley and Yong Sun Shim, Singular integrals on $C_{p}^{\alpha}$, Studia Math. 92 (1989), 285-293.

Department of Mathematics, University of Alberta, Edmonton, Alberta, Canada T6G 2G1 